\title{
Manque de compétences dans les soins de l'appareil locomoteur
}

\section{Bernhard Sorg}

Dr méd., membre de la FMH, 1951, 33 ans de pratique, anc. spécialiste en médecine générale, spécialiste en médecine interne générale depuis 2 ans

Dans son numéro du $1^{\text {er }}$ décembre 2017, la NZZ publiait sous le titre «Junge Ärzte sollen gipsen und schienen lernen» («Les jeunes médecins doivent apprendre à poser plâtres et attelles»)* un article sur un sujet qui préoccupe depuis longtemps les médecins de famille de l'ancienne génération dont je fais partie. Il n'a pourtant guère suscité de débat. Sans doute ne veut-on plus s'exposer ou se fatiguer davantage à dire ce qu'il faudrait changer quand rien ne changera de toute façon. «La pratique consistante à hospitaliser un skieur accidenté, même pour des blessures légères, est de plus en plus fréquente», y lit-on. "Les sociétés de médecine et la SUVA encouragent les médecins de famille des régions de montagne à compléter leur formation.» En effet, constate la NZZ, «depuis peu, les médecins ne sont plus tenus d'acquérir des connaissances en chirurgie des accidents dans le cadre de leur formation de base», cette discipline n'étant plus obligatoire. Toujours selon la NZZ, la SSTMA (Société Suisse de Traumatologie et de Médecine des assurances) compte organiser des cours spéciaux dès l'année à venir. Les jeunes médecins doivent apprendre à évaluer des radiographies et poser des plâtres et des attelles. Cette campagne est soutenue par la SUVA.

\section{Du généraliste à l'interniste généraliste}

Depuis la fusion de la SSMG et de la SSMI, le médecin de famille n'est plus un simple généraliste, mais littéralement un interniste généraliste. Nos futurs collègues se voient imposer un plan de formation qui ne reflète pas la médecine de famille actuelle. La formation obligatoire sur l'appareil locomoteur a été supprimée. On ne demande plus aux candidats ni chirurgie, ni traumatologie, ni orthopédie, ni rhumatologie, ni formation complémentaire en médecine manuelle (qui serait aussi plus que souhaitable), mais au moins trois ans de médecine interne.

Or, comment se présente en moyenne la pratique quotidienne d'un cabinet de premier recours? Au moins 33-66\% (voire jusqu'à 80\%) des consultations ont lieu pour des problèmes de l'appareil locomoteur à tous les niveaux: lombalgies, douleurs nucales ou à l'épaule, lésions de la hanche/du bassin, du genou, de la che- ville ou du pied, qu'elles soient d'origine traumatique, inflammatoire ou dégénérative. Comment le jeune médecin peut-il se retrouver dans cette jungle si on ne lui a pas fourni de boussole? Le fait que la SUVA (tout comme les autres assureurs privés LAA!) reconnaisse déjà cette insuffisance chez les fournisseurs de prestations avant même que la jeune génération ait pris la relève devrait faire réfléchir.

Cette crainte de poser un diagnostic ou d'instaurer un traitement sans support instrumental coûteux, je la rencontre chez de nombreux assistants de ma consultation. Il règne une grande insécurité. La décision de conseiller un traitement chirurgical ou conservateur est déléguée au spécialiste. Le généraliste n'a pas les connaissances nécessaires à une prise en charge faite d'attente et d'observation. Il connaît en revanche des syndromes exotiques qu'il ne rencontrera qu'une fois, sinon jamais, en 30 ans de carrière. Le nombre de cas vraiment problématiques de médecine interne est bien inférieur à un sur dix.

Une formation plus complète sur l'appareil locomoteur nous permettrait non seulement de tenir le niveau de prestations que nous devons à nos patients de médecine (interne) générale, mais aussi de réduire les frais encourus par les assureurs (longues incapacités de travail, clarifications coûteuses, transferts à l'hôpital, interventions chirurgicales inutiles, traitements interminables). Le réflexe de prescrire Novalgin et Dafalgan (parfois même avec un IPP) ne suffit pas à un traitement efficace de l'appareil locomoteur. Le médecin doit apprendre et appliquer les techniques d'injection, élargir ses connaissances des options médicamenteuses et se donner la possibilité d'évaluer correctement la capacité de travail en adéquation au profil professionnel du patient.

L'encouragement de la relève implique aussi de rendre celle-ci attentive aux défis qui l'attendent. Par grand bonheur, nos experts en formation ont au moins reconnu l'assistanat médical d'un an en cabinet de médecin de famille comme une formation à suivre. Il faudrait l'étendre à deux ans au début du plan de formation pour que les jeunes collègues sachent à quoi s'attendre et pourquoi choisir tel lieu de formation dans le but de pratiquer plus tard une médecine répondant à tous les contrôles et critères de qualité. 